\title{
On Characterization of Optimal Control Model of Whooping Cough
}

\author{
A. S. Ismail ${ }^{1}$ and Y. O. Aderinto ${ }^{2, *}$ \\ Mathematics Department, Faculty of Physical Sciences, University of Ilorin, P.M.B. 1515, Nigeria \\ e-mail: ahmed.ismail.senior@gmail.com ${ }^{1}$ \\ e-mail: aderinto@unilorin.edu.ng ${ }^{2}$
}

\begin{abstract}
Whooping cough is a vaccine avoidable public health problem which is caused by bacterium Bordetella Pertussis and it is a highly contagious disease of the respiratory system. In this paper, an SIR epidemiological model of whooping cough with optimal control strategy was formulated to control the transmission. The model was characterized to obtain the disease free and the endemic equilibrium points. Finally, the simulation was carried out using the Forward-backward sweep method by incorporating the Runge Kutta method to check the validity and the result obtained was an improvement over the existing results.
\end{abstract}

\section{Introduction}

Pertussis commonly known as whooping cough is one of the highly communicable bacterial respiratory tract infections mainly caused by Bordetella Pertussis. It is a major global health problem characterized by a paroxysmal cough.

Whooping cough affect all ages especially children and has caused more death than measles and polio combined. As one of the most deadly infectious diseases known worldwide, it is both an endemic and epidemic disease, responsible for significant morbidity and mortality especially in infants and children. WHO estimated 40 to 50 million cases of whooping cough and approximately 297,000 to 409,000 deaths annually where $90 \%$ cases belong to low income countries, [2], [7].

Vaccination with DTP (Diphtheria-tetanus-pertussis) has largely reduced the burden

Received: November 8, 2021; Accepted: December 4, 2021

2020 Mathematics Subject Classification: 49-XX, 49J15, 49K15, 49Mxx.

Keywords and phrases: whooping cough, model, characterization, equilibrium point, reproductive number. 
of this disease globally. However the number of adult cases have increased over the last decade and despite mass vaccination campaigns for over 50 years, and it is still increasing in developing countries, [9].

The disease typically lasts for approximately 6 to 10 weeks and the symptoms are more severe in infants or in individuals who have never been immunized against the disease. It is recognized with three stages: catarrhal, paroxysmal, and convalescent, [2]. The symptoms of the catarrhal stage are mild and may go unnoticed. The paroxysmal stage of Pertussis is characterized by episodes of coughing with a distinctive "whooping" sound when breathing in. This characteristic cough gives the disease its common name, Whooping Cough. During the convalescent stage, episodes of coughing are less frequent and symptoms improve. There is no seasonal pattern to outbreaks of this disease.

Historically, mothers have been the most common source of transmission of pertussis to their infants, and recent studies shows that mothers are the sources of $32 \%$ of the cases and other family members have up to $43 \%$ source of transmission of the disease to infants. Human infection with Bordetelle Pertussis depends on age and host immumity. Infants, especially those born prematurely and patients with underlying cardiac, pulmonary, neuromuscular, or neurologic disease are at high risk of contracting the disease. Its clinical signs include hypoglycemia, reduced pulmonary capacity and high leukocytosis. The complications include convulsions, bronchopneumonia, encephalopathy, apnoea, cyanosis, pneumoria and seizures, [3].

Recently, there has been a resurgence of whooping cough outbreak and western countries are highly endemic despite high vaccination coverage of about $90 \%$. Perhaps this could be that the vaccinated individuals can still become infected as a result of the disease mutation. The resurgence of whooping cough (pertussis) globally most especially in western countries with high vaccination coverage has been a concern. Hence, this work is based on optimal control of whooping model with two controls namely, vaccination and syndromic surveillance.

Mathematical models are considered to be important tools in analyzing the prevalence and control of infectious disease. Several works have been done on epidemics models by applying optimal control theory to SIR model using Pontryagin's Maximum Principle, [1]. [8] studied the optimal control treatment model of Hepatitis B Virus Dynamics. [5] worked on optimal control of nipah virus (NiV) infections. [12] investigate optimal control strategies for preventing hepatitis B infection and reducing chronic liver cirrhosis incidence. [6] studied the optimal control strategy for the immunotherapeutic 
treatment of HIV infection with state constraints. [4] modelled vacine distribution in rabies metapopulation and optimally controled it, to mention a few, [1], [5], [8], [12], [6], [4].

However, little or less has been done on optimal control of whooping cough with control, hence this work is about optimal control of whooping cough model with vaccination and syndromic surveillance as controls. The fundamental objective of syndromic surveillance is to reduce the contact rate between susceptible and infectious individuals. The optimal control strategy was developed and characterized to minimize the infected class, simulation was carried out using the Forward-backward sweep method by incorporating RK-4 method.

\section{Materials and Methods}

\subsection{Model formulation}

In this work, a mathematical SIR model for whooping cough transmission is considered. The whole population in model is divided into three: $S(t)$ is the susceptible population, $I(t)$ is infected population and $R(t)$ is recovered population. The following assumptions are imposed;

i. The population is fixed and all births are into susceptible class.

ii. The death rate is equal for all the three classes and the birth and death rates are equal so that the total population $N$ is stationary.

iii. $N$ is the fixed total population, normalizing to unity, i.e., $S(t)+I(t)+R(t)=1$.

Hence, the dynamic of whooping cough transimissionis formulated as model (A):

$$
\begin{gathered}
\frac{d S}{d t}=\omega-\omega S(t)-N \beta S(t) I(t) \\
\frac{d I}{d t}=N \beta S(t) I(t)-(\omega+\gamma) I(t) \\
\frac{d R}{d t}=\gamma I(t)-\omega R(t)
\end{gathered}
$$

where

$$
\begin{gathered}
S(0)=S_{0}, I(0)=I_{0} \text { and } R(0)=R_{0}, \\
S(0) \geq 0, I(0) \geq 0 \text { and } R(0) \geq 0 .
\end{gathered}
$$


The parameters used with their definitions are presented in Table 1.

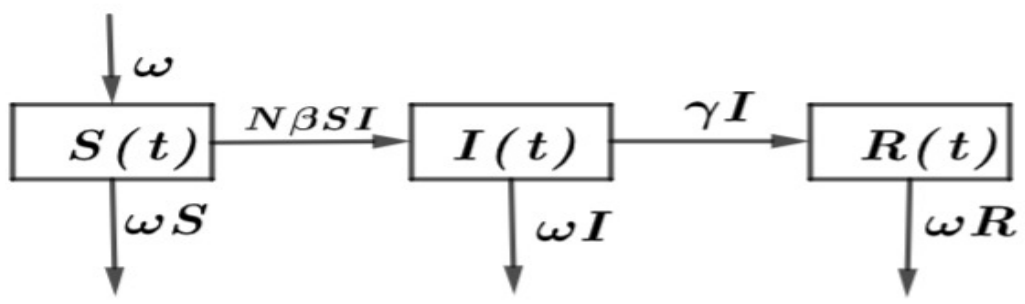

Figure 1: The flow diagram for the SIR model of whooping cough.

Table 1: Parameters and Definitions.

\begin{tabular}{|c|l|}
\hline Parameters & Definitions \\
\hline$\beta$ & Transmission rate coefficient \\
\hline$\gamma$ & Recovery rate \\
\hline$\omega$ & Birth rate / Death rate \\
\hline$N$ & Total population \\
\hline$I(t)$ & infected population \\
\hline$R(t)$ & recovered population \\
\hline$S(t)$ & susceptible population \\
\hline
\end{tabular}

\section{Characterization of the Model}

The model is characterized by investigating the equilibrium points, reproduction number, feasibility and boundedness of the solution.

\subsection{Equilibrium point}

Two equilibrium points was considered namely; The Disease Free Equilibrium Point $(I=0)$ and the Endemic Equilibrium Point $(I \neq 0)$.

At equilibrium the non-linear system (1)-(3) becomes;

$$
\begin{gathered}
\omega-\omega S(t)-N \beta S(t) I(t)=0 \\
N \beta S(t) I(t)-(\omega+\gamma) I(t)=0 \\
\gamma I(t)-\omega R(t)=0 .
\end{gathered}
$$




\section{The Disease Free Equilibrium (DFE) Point}

Let define the disease class as the population that are infectious. In the absence of the disease $I=0$, then equations (4) and (5) reduces to;

$$
\omega-\omega S=0 .
$$

Thus, $S^{D}=1$. Since $R=N-S-I$, thus $R=0$. Hence $D F E=\left(S^{D}, I^{D}, R^{D}\right)=(1,0,0)$ for $N=1$.

\section{The Endemic Equilibrium Point (EEP)}

The Endemic equilibrium point is the steady state solution where the disease persists in the population.

From equation (5)

$$
\begin{gathered}
N \beta S(t) I(t)-(\omega+\gamma) I(t)=0 \\
{[N \beta S(t)-(\omega+\gamma)] I(t)=0}
\end{gathered}
$$

thus;

$$
I \neq 0 \quad \text { or } \quad N \beta S-(\omega+\gamma)=0 .
$$

If

$$
N \beta S-(\omega+\gamma)=0 .
$$

It implies that;

$$
S^{E}=\frac{(\omega+\gamma)}{\beta} \quad \text { for } \quad N=1
$$

Substitute $S^{E}=\frac{(\omega+\gamma)}{N \beta}$ into equation (4) to obtain $I^{E}$,

$$
\omega-\omega \frac{(\omega+\gamma)}{N \beta}-N \beta\left(\frac{\omega+\gamma}{N \beta}\right) I=0
$$

thus;

$$
I^{E}=\frac{\omega}{\omega+\gamma}\left(1-\frac{9 \omega+\gamma)}{N \beta}\right)
$$

And since

$$
R=N-S-I
$$




$$
\begin{gathered}
R=N-\frac{\omega+\gamma}{N \beta}-\omega\left(\frac{N \beta-(\omega+\gamma)}{N \beta(\omega+\gamma)}\right) \\
R^{E}=\frac{\gamma}{\omega+\gamma}\left(1-\frac{(\omega+\gamma)}{N \beta}\right) .
\end{gathered}
$$

Hence, the endemic equilibrium point is given by;

$$
E E P=\left(S^{E}, I^{E}, R^{E}\right)=\frac{(\omega+\gamma)}{N \beta}, \frac{\omega}{\omega+\gamma}\left(1-\frac{(\omega+\gamma)}{N \beta}\right), \frac{\gamma}{\omega+\gamma}\left(1-\frac{(\omega+\gamma)}{N \beta}\right) .
$$

\subsection{The basic reproduction number $\left(R_{0}\right)$}

The basic reproduction number can be defined as the average number of secondary infections that occur when one infectious individual is introduced into a susceptible population with no immunity to the disease, in the absence of intervention. Therefore, when the basic reproduction number is less than 1 (i.e. $R_{0}<1$ ) then, the disease free equilibrium (DFE) is locally asymptotically stable while the endemic equilibrium is unstable, which mean that, at that point the disease dies out. But when $R_{0}>1$, it follows that an infectious individual will cause more than one additional infection on average, and thus, the disease will spread and the endemic equilibrium point is stable as a result of this. Whereas, when $R_{0}=1$, the disease becomes endemic (the disease remains in the population at a constant rate).

In order to determine the basic reproduction number, consider (2);

$$
\text { i.e. } \quad \begin{aligned}
\frac{d I}{d t} & =N \beta S(t) I(t)-(\omega+\gamma) I(t) \\
\frac{d I}{d t} & =[N \beta S(t)-(\omega+\gamma)] I(t) .
\end{aligned}
$$

Thus,

$$
\begin{aligned}
\int \frac{d I}{I} & =\int[N \beta S(t)-(\omega+\gamma)] d t \\
\ln I(t) & =[N \beta S(t)-(\omega+\gamma)] t+C
\end{aligned}
$$

with initial condition $t=0$ and $I(0)=I_{0}$ we have;

$$
I(t)=I_{0} e^{[N \beta S-(\omega+\gamma)] t} .
$$

Hence, the endemic equilibrium point occur when; 


$$
\begin{gathered}
N \beta S-(\omega+\gamma)>0 \\
N \beta S>(\omega+\gamma)
\end{gathered}
$$

therefore,

$$
\frac{N \beta S}{\omega+\gamma}>1
$$

Hence,

$$
R_{0}=\frac{N \beta}{(\omega+\gamma)} \quad \text { for } \quad S=1
$$

Therefore, the Endemic Equilibrium Point can be written in term of the reproduction number $R_{0}$ as;

$$
D F E=\left(S^{E}, I^{E}, R^{E}\right)=\frac{1}{R_{0}}, \frac{\omega}{\omega+\gamma}\left(1-\frac{1}{R_{0}}\right), \frac{\gamma}{(\omega+\gamma)}\left(1-\frac{1}{R_{0}}\right)
$$

\subsection{Optimal control of pertussis model dynamics}

Two controls namely vaccination and syndromic surveillance were incorporated into the model A to have model B as;

$$
\begin{gathered}
\frac{d S}{d t}=\omega-\omega S(t)-\left(1-u_{2}\right) N \beta S(t) I(t)-u_{1} S(t) \\
\frac{d I}{d t}=\left(1-u_{2}\right) N \beta S(t) I(t)-(\omega+\gamma) I(t) \\
\frac{d R}{d t}=\gamma I(t)-\omega R(t)+u_{1} S(t)
\end{gathered}
$$

where $u_{1}$ is a fraction of susceptible vaccinated per unit time and $u_{2}$ is a reduction in contact rate due to surveillance.The total population size $N=S(t)+I(t)+R(t)$, implies that;

$$
\frac{d N}{d t}=\omega-\omega N
$$

\section{Optimality of the System}

Optimal control problem involving two controls $u_{1}(t)$ and $u_{2}(t)$ was developed. Where $u_{1}(t) S(t)$ is some amount of susceptible individuals that are vaccinated and 
$u_{2}(t) N \beta S(t) I(t)$ is the reduction in contact rate between the susceptible and Infected individuals. It is assumed that all susceptible population that is vaccinated is directly transferred to recovered class. The objective is to minimize infected individuals, hence we seek an optimal control strategy that minimizes the infected population and the cost associated with controls. Let $k$ be a positive constant, $z_{1}$ and $z_{2}$ be weights factors act as cost associated with controls $u_{1}$ and $u_{2}$.

Hence the problem is;

Minimize $\quad J\left(u_{1}, u_{2}\right)=\int_{0}^{T_{f}}\left[K I(t)+\frac{1}{2}\left[z_{1} u_{1}^{2}(t)+z_{2} u_{2}^{2}(t)\right]\right]$

Subject to

$$
\begin{gathered}
\frac{d s}{d t}=\omega-w s(t)-\left(1-u_{2}\right) N \beta S I-u_{1} S \\
\left.\frac{d I}{d t}=\left(1-u_{2}\right) N \beta S I-(\omega+\gamma) I\right) \\
\frac{d R}{d t}=\gamma I-\omega R+u_{1} S
\end{gathered}
$$

where

$$
\begin{gathered}
S(0) \geq 0, \quad I(0) \geq 0, \quad R \geq 0 \\
S\left(T_{f}\right)=I\left(T_{f}\right)=R\left(T_{f}\right)=\text { free } .
\end{gathered}
$$

Let

$$
L\left(I, u_{1}, u_{2}\right)=K I(t)+\frac{1}{2}\left[z_{1} u_{1}^{2}(t)+z_{2} u_{2}^{2}(t)\right]
$$

where $L$ is the lagrangian and the Hamiltonian of the system is given as

$$
H\left(I, u_{1}, u_{2}, \lambda_{s}, \lambda_{I}, \lambda_{R}\right)=L+\lambda_{s}(t) S^{\prime}(t)+\lambda_{I}(t) I^{\prime}(t)+\lambda_{R} R^{\prime}(t)
$$

where $\lambda_{s}, \lambda_{I}, \lambda_{R}$ are adjoint variables

$$
H\left(I, u_{1}, u_{2}, \lambda_{s}, \lambda_{I}, \lambda_{R}\right)=K I(t)+\frac{1}{2}\left[z_{1} u_{1}^{2}(t)+z_{2} u_{2}^{2}(t)\right]+F_{1}+F_{2}+F_{3}
$$

where

$$
\begin{gathered}
F_{1}=\lambda_{S}(t)\left(\omega-w s(t)-\left(1-u_{2}\right) N \beta S I-u_{1} S\right), \\
F_{2}=\lambda_{I}(t)\left(\left(1-u_{2}\right) N \beta S I-(\omega+\gamma) I\right),
\end{gathered}
$$




$$
F_{3}=\lambda_{R}(t)\left(\gamma I-\omega R+u_{1} S\right)
$$

The associated adjoints equations are;

$$
\begin{gathered}
\lambda_{S}{ }^{\prime}=-\left(\frac{\delta H}{\delta S}\right)=\lambda_{S}\left(\omega+\left(1-u_{2}\right) N \beta I+u_{1}\right)-\lambda_{I}\left(1-u_{2}\right) N \beta I-\lambda_{R} u_{1} \\
\lambda_{I}{ }^{\prime}=-\left(\frac{\delta H}{\delta I}\right)=-\left[k-\lambda_{S}\left(1-u_{2}\right) N \beta S+\lambda_{I}\left[\left(1-u_{2}\right) N \beta S-(\omega+\gamma)\right]+\lambda_{R} \gamma\right] \\
\lambda_{R}{ }^{\prime}(t)=-\left(\frac{\delta H}{\delta R}\right)=\lambda_{R} \omega .
\end{gathered}
$$

Transversality conditions

$$
\lambda_{S}\left(T_{f}\right)=\lambda_{I}\left(T_{f}\right)=\lambda_{R}\left(T_{f}\right)=0 .
$$

The optimality conditions of the system are;

$$
\frac{\delta H}{\delta u_{1}}=0, \quad u_{1}^{\prime}=\frac{\left(\lambda_{S}-\lambda_{R}\right) S}{z_{1}}
$$

and

$$
\frac{\delta H}{\delta u_{2}}=0, \quad u_{2}^{\prime}=\frac{\left(\lambda_{I}-\lambda_{S}\right)}{z_{2}} N \beta S I .
$$

Thus $\left(X^{*}, u_{1}^{*}, u_{2}^{*}\right)$ is an optimal solution of the optimal control problem for $X=(S, I, R)$, $0 \leq u_{i} \leq 1, i=1,2$.

Thus

$$
u_{1}^{*}= \begin{cases}0 & \text { if } m_{1}^{*} \leq 0 \\ m_{1}^{*} & \text { if } 0<m_{1}^{*}<1 \\ 1 & \text { if } m_{1}^{*} \geq 1\end{cases}
$$

where $m_{1}^{*}=\frac{\left(\lambda_{S}-\lambda_{R}\right) S}{z_{1}}$

and

$$
u_{2}^{*}= \begin{cases}0 & \text { if } m_{2}^{*} \leq 0 \\ m_{2}^{*} & \text { if } 0<m_{2}^{*}<1 \\ 1 & \text { if } m_{2}^{*} \geq 1\end{cases}
$$

where $m_{2}^{*}=\frac{\left(\lambda_{I}-\lambda_{S}\right) N \beta S I}{z_{2}}$. 


\section{Numerical Implication}

In this section, numerical simulations of the whooping cough model was described for more understanding of the system. The concept of forward-backward sweep method, [10] was used to solve the optimality system consisting of six (6) ordinary differential equations arriving from the state and adjoint equations coupled with the two controls. The parameters used for simulationswere chosen based on literature, [11].

\subsection{Forward-backward sweep method}

To solve an optimal control problem

$$
J=\min _{(u)} \int_{t_{0}}^{t_{1}} f(t, x(t), u(t)) d t
$$

Subject to

$$
\dot{x}(t)=g(t, x(t), u(t))
$$

and $x\left(t_{0}\right)=a$

$$
\begin{aligned}
& \dot{\lambda}(t)=-\left(\frac{\partial H}{\partial x}\right)=-\left(f_{x}(t, x, u)+\lambda(t)\left(g_{x}(t, x, u)\right)\right) \\
& \lambda\left(t_{1}\right)=0 \\
& 0=\frac{\partial H}{\partial u}=\left(f_{u=u^{*}}(t, x, u)\right)+\lambda(t)\left(g_{u=u^{*}}(t, x, u)\right),
\end{aligned}
$$

where last equation is employed to find $u^{*}$ in terms of $t$ and $x$ and $\lambda$ and when this representation is substituted back into the ODEs for $x$ and $\lambda$ the above two equations form a two-point boundary value problem, we partition the time interval $\left[t_{0}, t_{1}\right]$ into usually equally spaced points $t_{0}=b_{1}, b_{2}, b_{N}, b_{N+1}=t_{1}$. The approximation will be a vector $\vec{u}=\left(u_{1}, u_{2}, u_{3}, u_{N+1}\right)$, where $u_{i} \approx u\left(b_{i}\right)$.

\subsection{Simulation of whooping cough model}

The simulation of the whooping cough model was carried out and the results was shown graphically in Figures 5.1-5.4 using $h=0.01, \omega=0.04, \gamma=24$ and $N \beta=$ 123. $S(0)=0.24, I(0)=0.007$ and $R(0)=0.753$, some of the parameter values were from [11]. 


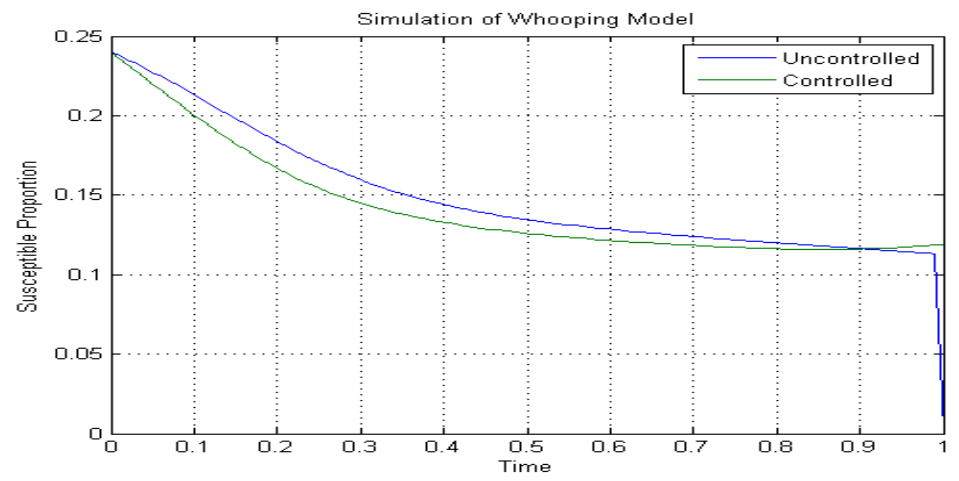

Figure 5.1: The Susceptible proportion of whooping cough model versus Time with and without controls.

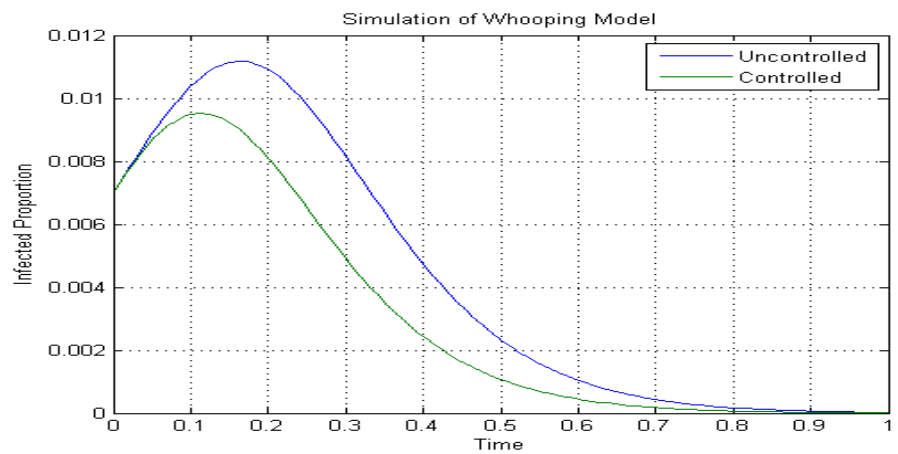

Figure 5.2: The Infected proportion of whooping cough model versus Time with and without controls.

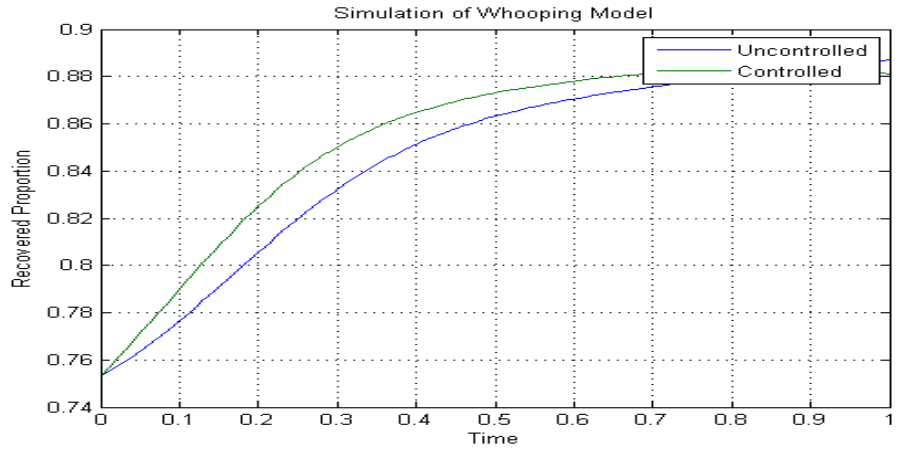

Figure 5.3: The Recovered proportion of whooping cough model versus Time with and without controls. 


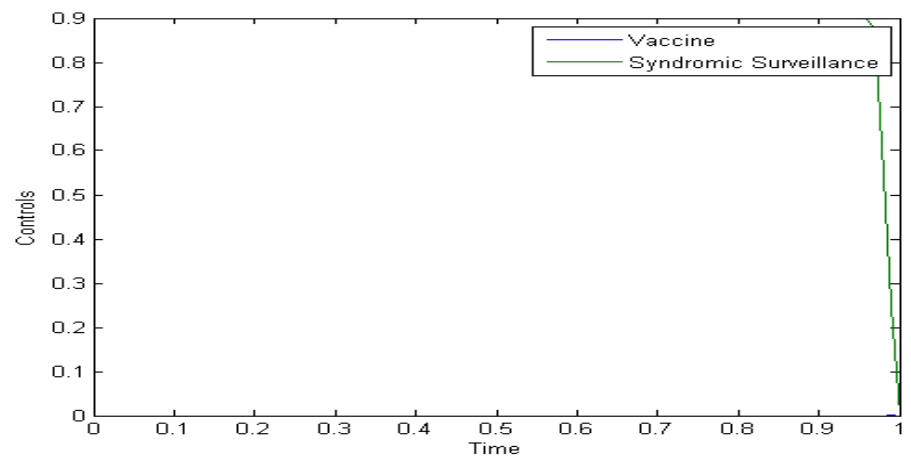

Figure 5.4: The optimal control model of whooping cough.

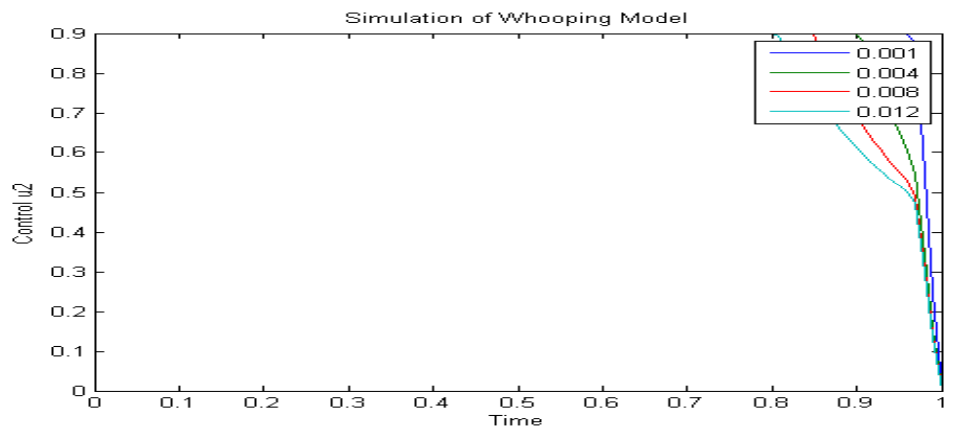

Figure 5.5: The optimal control model versus Time by varying the weight factors $z_{2}$.

\subsection{Interpretation of results}

The results obatined from the simulation show that the infected population can be reduced significantly in the presence of control, Figures 5.1-5.5.

Figure 5.1 shows the simulation of the susceptible proportion versus time, susceptible population is decreased with application of control. Susceptible population without control was represented by blue line while with control was represented by green line.

Figure 5.2 shows the simulation of the infected population versus time. Infected population is also significantly reduced with the application of optimal control.

Figure 5.3 shows the simulation of the recovered population versus time. Recovered population with optimal control is increased.

Figure 5.4 represent the optimal control variables $u_{1}$ and $u_{2}$ at time $t$ which play a significant role in minimizing the infected population in the host population. 
Figure 5.5 shows the simulation of the control versus time but varied values of weight factor $z_{2}$ through the values $0.001,0.004,0.008$ and 0.012. Simulations reveal that when we increase the weight factor $z_{2}$ the amount of the control decreases.

\section{Conclusion}

In this work, optimal control model of whooping cough was characterized in an attempt to minimize the infected population. An optimal control strategy was developed, simulations of the optimal control problem demonstrates that under the impact of external controls susceptible and infected populations are decreased while recovered population increased. Thus pertussis disease can be minimized through the introduction of the optimal control stategies.

\section{References}

[1] A.J. Arenas, G. Gonzalez-Parra and B.M. Chen-Charpentier, A nonstandard numerical scheme of predictor-corrector type for epidemic models, Computers and Mathematics with Applications 59(12) (2010), 3740-3749. https://doi:10.1016/j.camwa.2010.04.006

[2] E.L. Arnal, T. Grunert, N. Cattelan, D. Degouw, M.I. Villalba, D.O. Serra, F.R. Mooi, M.E. Ehling-Schulz and O.M. Yamtorno, Bordetella pertussis isolates from argentinean whooping cough patients display enhanced biofilm formation capacity compared to Tohama I reference strain, Frontiers in Microbiology 1352 (2015), 1-12. https://doi.org/10.3389/fmicb.2015.01352

[3] A. Aslanabadi, K. Ghabili, K. Shad, M. Khalili and M. Sajadi, Emergence of whooping cough: notes from three early epidemics in Persia, The Lancet Infectious Disease 15(12) (2015), 1480-1484. https://doi.org/10.1016/s1473-3099(15)00292-3

[4] E. Asano, L.J. Gross, S. Lenhart and L.A. Real, Optimal control of vaccine distribution in a rabies metapopulation model, Journal of Mathematical Biosciences and Engineering 1 (2007), 1-20.

[5] M.H.A. Biswas, Optimal control of nipah virus (NIV) infections: A Bangladesh scenario, Journal of Pure and Applied Mathematics: Advances and Applications 12(1) (2014), 77104.

[6] M.H.A. Biswas, M.M. Haque and U.K. Mallick, Optimal control strategy for the immunotherapeutic treatment of HIV infection with state constraint, Optimal Control Applications and Methods 40(4) (2019), 807-818. https://doi.org/10.1002/oca.2516

[7] V. Bouchez and N. Guiso, Bordetella pertussis, B. parapertussis, vaccines and cycles of whooping cough, Pathogens and Disease 73(7) (2015), 01-06.

https://doi.org/10.1093/femspd/ftv055 
[8] A.M. Elaiw, M.A. Alghamdi and S. Aly, Hepatitis B virus dynamics; modeling, analysis and optimal treatment scheduling, Discrete Dynamics in Nature and Society 2013 (2013), 21-29. https://doi.org/10.1155/2013/712829

[9] J.S. Lavine, A.A. King and O.N. Bjørnstad, Natural immune boosting in pertussis dynamics and the potential for long-term vaccine failure, Proceedings of the National Academy of Sciences 108(17) (2011), 7259-7274.

https://doi.org/10.1073/pnas.1014394108

[10] S. Lenhart and J.T. Workman, Optimal control applied to biological models, Mathematical and Computational Biology Series, Chapman and Hall/CRC, London, 2007.

[11] G.G. Parra, A.J. Arenas and B.M. Chen-Charpentier, Combination of nonstandard schemes and Richardson's extrapolation to improve the numerical solution of population models, Mathematical and Computer Modeling 52 (2010), 1030-1036. https://doi.org/10.1016/J.MCM.2010.03.015

[12] Mst. Shanta Khatun and Md. Haider Ali Biswas, Optimal control strategies for preventing hepatitis B infection and reducing chronic liver cirrhosis incidence, Infectious Disease Modelling 5 (2020), 91-110. https://doi.org/10.1016/j.idm.2019.12.006

This is an open access article distributed under the terms of the Creative Commons Attribution License (http://creativecommons.org/licenses/by/4.0/), which permits unrestricted, use, distribution and reproduction in any medium, or format for any purpose, even commercially provided the work is properly cited. 